\title{
The Association Between the Severity of the Mandibular Inferior Cortex Porosity and the Number of Remaining Teeth in Elderly Indonesian People
}

Bramma Kiswanjaya ( $\sim$ brammakiswanjaya@gmail.com )

Faculty of Dentistry, Universitas Indonesia https://orcid.org/0000-0002-9856-1724

Hanna H Bachtiar-Iskandar

Faculty of Dentistry, Universitas Indonesia

Research article

Keywords: Mandibular bone, panoramic radiographs, elderly people, osteoporosis

Posted Date: September 4th, 2019

DOI: https://doi.org/10.21203/rs.2.13979/v1

License: (c) (1) This work is licensed under a Creative Commons Attribution 4.0 International License.

Read Full License 


\section{Abstract}

Background The purpose of this study was to investigate the association between the severity of the mandibular inferior cortex (MIC) porosity and the number of remaining teeth in elderly Indonesian people.

Materials and methods This cross-sectional study was conducted by measuring secondary data on panoramic digital examinations of patients aged 50 years and older. A total of 428 panoramic radiographs were measured from January 2017 to December 2018. The number of remaining teeth (including the third molars) was evaluated. Mandibular porosity was measured by using panoramic radiographs, according to the MIC classification: normal; mildly to moderately eroded; and severely eroded cortex.Statistical analysis used Data analysis was done using Mann-Whitney nonparametric tests to verify the relationship between the MIC classification and the number of remaining teeth. Multiple linear regression analysis was performed to investigate the relationship between confounding variables.

Results A significant correlation was found between MIC classification and the number of remaining teeth on the Mann-Whitney test $(P<0.001)$. Multiple linear regression analysis showed that MIC classification was significantly associated with the value of the number of remaining teeth after controlling for gender and age $(\beta=-0.37, \mathrm{R} 2=0.22, \mathrm{P}<0.001)$. Subjects with MIC class 3 were 7.14 times more likely to lose their teeth than subjects with MIC class 1 or class 2 .

Conclusions Our study demonstrated that there was an association between the severity of mandibular inferior cortex porosity and the number of remaining teeth in elderly Indonesian people.

\section{Background}

Tooth loss is associated with aesthetic, functional, psychological, and social impacts for elderly people. As tooth loss increases with age ${ }^{[1]}$ it has been accepted as a handicapping condition. However, elderly people who lose teeth are more likely to feel less confident, restrict food choice, enjoy food less, avoid laughing in public and avoid forming close relationships. ${ }^{[2]}$ The causes of tooth loss include periodontitis ${ }^{[3]}$ and unrestorable teeth or periapical lesions due to caries, ${ }^{[4]}$ all of which are easily detected by dentists. Tooth loss due to aging processes such as osteoporosis is more difficult to detect. Several studies conducted to determine the association between oral health and osteoporosis used diagnostic tools that were unfamiliar to dentists. ${ }^{\left[{ }^{[}\right],[6]}$ Research is needed to determine how dentists can contribute to osteoporosis detection and to investigate the relationship between the severity of jawbone porosity and the number of remaining teeth.

Mandibular inferior cortex (MIC) classification, as defined by Klemetti et al., ${ }^{[7]}$ is used to detect early osteoporosis with panoramic radiography. The relationship between the Klemetti index and heel bone mineral density (BMD) has been studied using a diagnostic tool for osteoporosis (dual energy X-ray absorptiometry), ${ }^{[8]}$ biochemical markers of bone metabolism, ${ }^{[9]}$ and mandibular bone quality. ${ }^{[10]}$ Our previous research found a positive relationship between MIC classification and bone stiffness on the os 
calcis $^{[11]}$, and an association with total serum calcium tracked using panoramic radiography in a longitudinal study. ${ }^{[12]}$

Although some investigators have demonstrated a significant association between jawbone condition and tooth loss, ${ }^{[13]}$ others have failed to find this association. ${ }^{[14],[15]}$ One study that measured the periodontal conditions of 210 females and compared an osteoporosis group to a control group with normal BMD found no significant difference in periodontal condition between the osteoporosis group and the control group, ${ }^{[14]}$ but the authors acknowledged that this may have been due to the small sample.

The studies mentioned were in developed countries with good oral health awareness and advanced dental health facilities. Studies with a high number of subjects in developing countries are needed to investigate whether the severity of mandibular bone porosity as a sign of osteoporosis is related to the number of remaining teeth. The purpose of this study was to investigate the association between the severity of the MIC porosity and the number of remaining teeth in elderly Indonesian people.

\section{Materials And Methods}

This cross-sectional study was based on secondary data from panoramic digital examinations of patients 50 years and older. The age of 50 was selected, as it has been reported that age-related loss of bone was more prominent in women after the age of 50 compared to those below $50 .{ }^{[16]}$ This study was approved by the Ethical Committee of the Faculty of Dentistry, Universitas Indonesia.

Panoramic radiographs from January 2017 to December 2018 were chosen. An initial 439 digital panoramic radiographs were collected from the radiology medical records of the study hospital. After evaluating the quality of the radiographs in the area to be interpreted, eight radiographs were found not to be in the panoramic standard head position. Incomplete variable data and radiographs that showed cysts involving the jaw, benign or malignant tumors, suspected or self-reported systemic conditions, and jaw fractures that affected the numbers of remaining teeth were not included in the study. For subjects who underwent panoramic radiographic examinations more than once during the 2017-2018 period, only the final radiographic examination was used if it met the inclusion criteria. A total of 428 subjects of panoramic radiographs were measured in the study. All subjects were Indonesian.

The numbers of remaining teeth (including the third molars) were evaluated on panoramic radiographs using the following categories: healthy, carious, or treated. Root tips indicated for extraction were not included as the remaining teeth. Measurement of the MIC was determined by observing the mandible distally from the mental foramen on both sides. Subjects were divided into three groups (C1-C3), according to the Klemetti method ${ }^{[7]}$ (Figure 1). $\mathrm{C} 1$ (normal cortex) indicated that the endosteal margin of the cortex was even and sharp on both sides, C2 (mildly to moderately eroded cortex) indicated that the endosteal margin showed semi-lunar defects (lacunar resorption) or appeared to form endosteal cortical residues on one or both sides, and C3 (severely eroded cortex) indicated that the cortical residues were clearly porous on one or both sides. 
All digital radiographs were taken with the Veraviewepocs 2D § (J. Morita, Kyoto, Japan). The Veraviewepocs $2 \mathrm{D} \circledast$ orthopantomograms were taken with a high-resolution Charge-Coupled Device (CCD) sensor (32-bit microprocessor) (J. Morita, Kyoto, Japan). The panoramic exposure was made at ten milliampere-seconds (mAs) for $12-15 \mathrm{~s}$ at $70-80 \mathrm{kVp}$. Radiographs were taken by the same radiographer. One well-trained examiner with ten years' experience determined the MIC classification. To calculate the intra- and inter-examiner agreement, a dentist was trained to measure MIC classification. After a period of calibration, 100 panoramic radiographs were chosen randomly and reanalyzed within a period of one week. The kappa agreement for intra- and inter-agreement was 0.88 and 0.80 , respectively. Based on the guidelines of the interpretation of kappa statistics performed by Landis and Koch, ${ }^{[17]}$ the following categories were considered: 0.00 (poor), $0.00-0.20$ (slight), $0.21-0.40$ (fair), $0.41-0.60$ (moderate), $0.61-0.80$ (substantial) and 0.81-1.00 (almost perfect). The kappa value of intra- and inter agreements was in the category of almost perfect and substantial, respectively.

\section{Statistical analysis}

The results of the MIC classification were divided into two groups. Group 1 comprised MIC class 1 and class 2 which are still considered normal. Group 2 consisted of MIC class 3 which is considered at high risk of osteoporosis. The homogeneity of the collected data was assessed, and a non-normal distribution was observed. Mann-Whitney nonparametric tests were used to verify the relationship between MIC classification and the number of remaining teeth. Finally, multiple linear regression analysis was used to assess the relationship between MIC classification and the number of remaining teeth after controlling for confounding factors. The number of remaining teeth was used as a dependent variable. MIC classification (C1 and C2:0; C3:1), age (group 1: 50-64 years old; group 2: 65-88 years old), gender (female:0; male:1) used as an independent variable. The level of significance was set at $P<0.05$ for these tests. Statistical analysis was performed using SPSS software for Windows, version 20.0 (SPSS, Inc, Chicago, IL, USA).

\section{Results}

The description of the 428 subjects is shown in Table 1. The mean age and number of remaining teeth were $59 \pm 7.2$ years old and $21 \pm 8.7$ teeth, respectively. The total subject percentages in Group 1 (MIC class 1 or class 2 ) were $72.2 \%$, and group 2 (MIC class 3 ) were $27.8 \%$. There were significant differences in gender, age and number of remaining teeth between group 1 and group 2 according to the Chi-square test.

The relationship between MIC classification and the number of remaining teeth is shown in Table 2 . The median of the number of remaining teeth in group 1 (MIC class 1 or class 2) and group 2 were 25 and 16 teeth, respectively. A significant correlation was found between MIC classification and the number of remaining teeth on the Mann-Whitney test $(P<0.001)$. 
The results of multiple linear regression analysis using the number of remaining teeth as a dependent variable are shown in Table 3. The MIC classification between group 1 and group 2 was significantly associated with the value of the number of remaining teeth after controlling for gender and age $(\beta=-$ $0.37, \mathrm{R} 2=0.22, P<0.001)$.

\section{Discussion}

Our study demonstrated that there was an association between the severity of mandibular inferior cortex porosity and the number of remaining teeth in elderly Indonesian people. Subjects in group 2 (MIC class 3) showed significantly fewer teeth than a subject in group 1 (MIC class 1 or class 2 ). These findings are supported by another study that suggested that mandibular inferior cortex porosity is associated with tooth loss. ${ }^{[18]}$ Osteoporosis may have an impact on these findings, and many studies have demonstrated a significant relationship between jawbone measurements and BMD at other skeletal sites. ${ }^{[7],[8],[9],[10],[11],}$ [12]

The region of interest in this research was the mandibular inferior cortex, which is not associated with local factors such as periodontitis or periapical lesions. The osteoporosis changes in the alveolar crest and jawbone could directly affect the retention and stability of the teeth. This suggests that the severity of osteoporosis is related to loss of height of the alveolar crest and tooth loss in elderly people. Previous research has demonstrated that osteoporosis affects the quality of jawbone, ${ }^{[19]}$ the severity of clinical attachment loss, ${ }^{[20]}$ and, in the end, the number of remaining teeth. Although osteoporosis was not the primary cause for losing teeth, it is a risk factor that may contribute to the progression of periodontal disease and the reduction of the alveolar bone crest. This study suggested that subjects considered at high risk of osteoporosis had a higher risk of losing teeth than subjects considered normal risk.

Research has shown that the long-term use of hormone therapy (10 years or more) shows an average decrease in tooth loss by three teeth, compared with those who did not use the therapy. ${ }^{[21]}$ Other investigators showed that supplementation with calcium and vitamin D significantly reduced tooth loss in the elderly over a period of three years, increased bone mineral density and decreased risk of tooth loss. ${ }^{[22]}$ These findings suggest that collaboration between medical and dental professionals is needed to prevent tooth loss related to osteoporosis. Dentists could screen patients at risk of osteoporosis using panoramic radiographs and refer patients to a medical professional to confirm the diagnosis of osteoporosis.

In this study, the subjects were divided into two categories based on a previous study that used means and standard deviations of bone stiffness by using ultrasound bone densitometry. The study showed that the Z-scores of bone stiffness among the MIC classifications class 1 and class 2 were still considered normal (Z-score $\geq 1 \mathrm{SD}$ ) and the MIC classification class 3 were considered at high risk for osteoporosis (Z-score $\leq-1.0 \mathrm{SD}) .{ }^{[11]}$ This grouping was carried out to investigate the condition of the jawbone that was at high risk of developing osteoporosis and its relation to the number of remaining teeth. These categories also avoid errors in analysing the MIC classification in dental clinic. For dentists who are not 
used to measuring MIC classification, distinguishing between a normal (Class 1) and a mildly eroded (Class 2) mandibular cortex is difficult.

The multiple linear regression demonstrated a significant association of the number of remaining teeth with a MIC classification group after controlling for the variables of age and gender. However, the R square indicated that those variables have less influence on the number of remaining teeth. Tooth loss is influenced more by local factors such as an untreated periapical lesion and periodontal disease. In this study design, it was difficult to know if the tooth loss presented was related to caries or periodontal disease as well as osteoporosis change of the mandible that contributed to increased risk of tooth loss. This result shows that other variables exist to explain the number of remaining teeth. Although other variables are needed to investigate the relationship in the number of remaining teeth, the result demonstrated that the number of remaining teeth was significantly decreased in group 2 with MIC class 3. Furthermore, subjects in group 2 with MIC class 3 were 7.14 times more likely to lose their teeth than subjects in group 1 with MIC class 1 or class 2 .

The study has some limitations. We used secondary data from the available panoramic radiographs. Therefore, the study had a cross-sectional design. A longitudinal study is needed to investigate the cause of tooth loss. Also, further research needs to be done to include local factors that caused tooth loss to investigate the relationship among those variables.

\section{Conclusions}

Our study demonstrated that there was an association between the severity of mandibular inferior cortex porosity and the number of remaining teeth in elderly Indonesian people. Collaboration between medical and dental professionals is needed to prevent tooth loss related to osteoporosis.

\section{Declarations}

\section{Ethics}

This study was approved by the Ethical Committee of the Faculty of Dentistry, Universitas Indonesia, letter number: 35/Ethical approval/FKGUI/III/2019

\section{Funding}

The publication of this manuscript is supported by the Directorate of Research and Community Service (DRPM), University of Indonesia from grant: Hibah QQ 2019, contract number: NKB0245/UN2.R3.1/HKP.05.00/2019

\section{Conflicts of interest}


The authors declare no potential conflicts of interest concerning the research, authorship, and publication of this article.

\section{References}

1. Linden GJ, Linden K, Yarnell J, Evans A, Kee F, Patterson CC. All-cause mortality and periodontitis in 60-70-year-old men: a prospective cohort study. J Clin Periodontol 2012;39:940-6. 2. Davis DM, Fiske J, Scout B, Radford DR. The emotional effects of tooth loss in a group of partially dentate people: a quantitative study. Eur J Prosthodont Restor Dent 2001; 9:53-7. 3. Armitage GC, Robertson PB. The biology, prevention, diagnosis and treatment of periodontal diseases: scientific advances in the United States. J Am Dent Assoc 2009; 140:36S-43S. 4. Dikbas I, Tanalp J, Tomruk CO, Koksal T. Evaluation of reasons for extraction of crowned teeth: a prospective study at a university clinic. Acta Odontol Scand 2013; 71:848-6. 5. Mohammad AR, Brunsvold M, Bauer R. The strength of association between systemic postmenopausal osteoporosis and periodontal disease. Int J Prosthodont 1996;9:479-3. 6. Jagelaviciene $\mathrm{E}$, Kubilius R. The relationship between general osteoporosis of the organism and periodontal diseases. Medicina (Kaunas) 2006;42:613-8. 7. Klemetti E, Kolmakov S, Kröger H. Pantomography in assessment of the osteoporosis risk group. Scand J Dent Res 1994;102:68-72. 8. Halling A, Persson GR, Berglund J, Johansson O, Renvert S. Comparison between the Klemetti index and heel DXA BMD measurements in the diagnosis of reduced skeletal bone mineral density in the elderly. Osteoporos Int 2005;16:999-1003. 9. Deguchi T, Yoshihara A, Hanada N, Miyazaki H. Relationship between mandibular inferior cortex and general bone metabolism in older adults. Osteoporos Int 2007;19:935-40. 10. Horner K, Devlin H. The relationships between two indices of mandibular bone quality and bone mineral density measured by dual energy X-ray absorptiometry. Dentomaxillofac Radiol 1998;27:17-21. 11. Kiswanjaya B, Yoshihara $A$, Deguchi T, Miyazaki H. Relationship between the mandibular inferior cortex and bone stiffness in elderly Japanese people. Osteoporosis Int 2010;21:433-8. 12. Kiswanjaya B, Yoshihara A, Miyazaki H. Mandibular inferior cortex erosion as a sign of elevated total serum calcium in elderly people: a 9-year follow-up study. Dentomaxillofac Radiol 2014;43:20130341. 13. Taguchi A, Tanimoto K, Suei Y, Wada T. Tooth loss and mandibular osteopenia. Oral Surg Oral Med Oral Pathol Oral Radiol Endod 1995;79:12732. 14. Lundström $\AA$, Jendle J, Stenström B, Toss G, Ravald N. Periodontal conditions in 70-year-old women with osteoporosis. Swed Dent J 2001;25:89-96. 15. Slaidina A, Soboleva U, Daukste I, Zvaigzne A, Lejnieks A. Postmenopausal osteoporosis and tooth loss. Stomatologija 2011;13:92-5. 16. López-Marcos JF, García-Valle S, García-Iglesias AA. Periodontal aspects in menopausal women undergoing hormone replacement therapy. Med Oral Patol Oral Cir Bucal 2005;10:132-41. 17. Landis JR, Koch GG. The measurement of observer agreement for categorical data. Biometrics 1977;33:159-74. 18. Yohihara A, Deguchi T, Miyazaki H. Relationship between bone fragility of mandibular inferior cortex and tooth loss related to periodontal disease in older people. Community Dent Health 2011;28:165-9. 19. Jeffcoat M K, Lewis C E, Reddy M S, Wang C Y, Redford M. Post-menopausal bone loss and its relationship to oral bone loss. Periodontol 2000;23: 94-102. 20. Genco R J, Grossi S G. Is estrogen deficiency a risk factor for periodontal disease? Compend Contin Educ Dent Suppl; 1998.S23-9. 21. Taguchi A, Kobayashi J, Suei Y, Ohtsuka M, Nakamoto T, Tanimoto $\mathrm{K}$ et al. Association of estrogen and vitamin $\mathrm{D}$ receptor gene 
polymorphisms with tooth loss and oral bone loss in Japanese postmenopausal women. Menopause 2003;10:250-7 22. Dietrich T, Joshipura KJ, Dawson-Hughes B, Bischoff-Ferrari HA. Association between serum concentrations of 25-hydroxyvitamin D3 and periodontal disease in the US population. Am J Clin Nutr 2004;80:108-13.

\section{Tables}

Due to technical limitations, tables are only available as a download in the supplemental files section

\section{Figures}

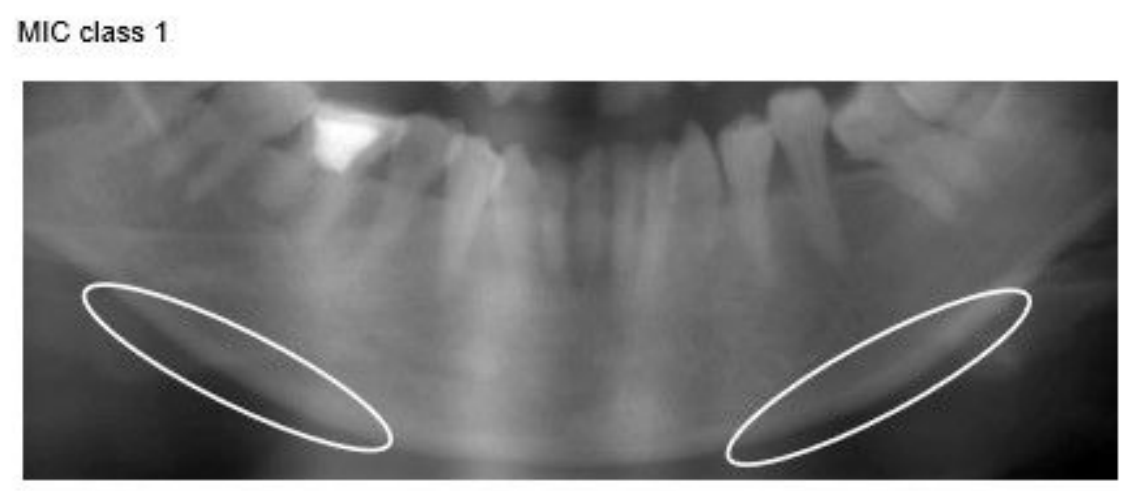

MIC class 2

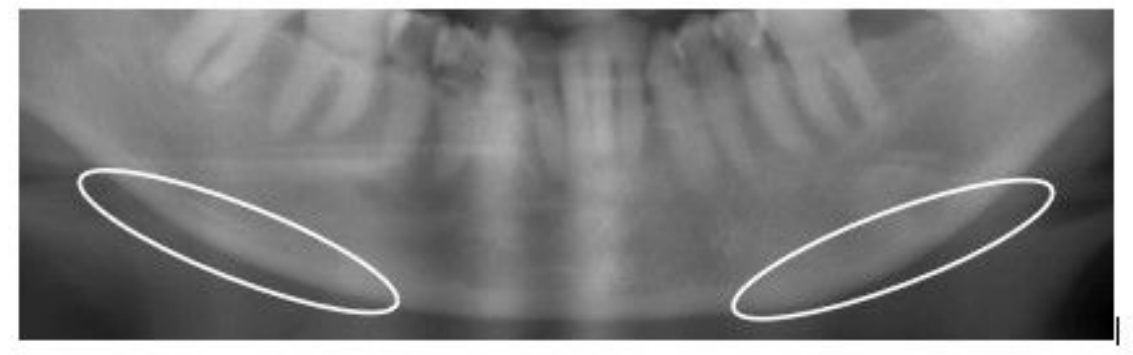

MIC class 3

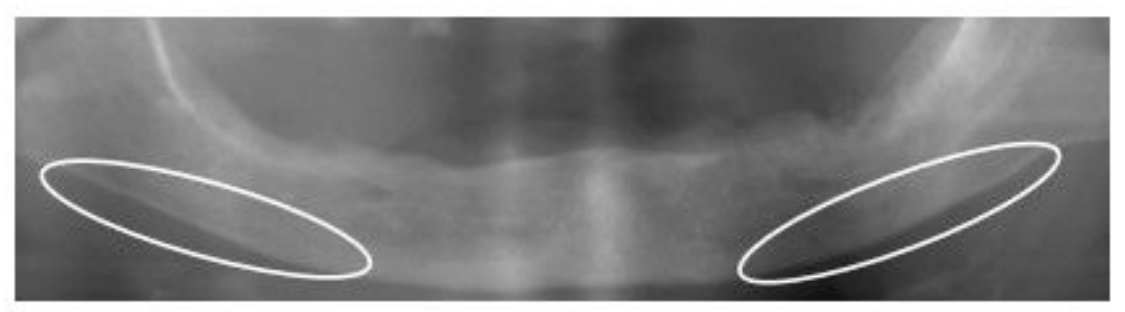

Figure 1

Mandibular Inferior Classification (MIC) 


\section{Supplementary Files}

This is a list of supplementary files associated with this preprint. Click to download.

- Table3.Theresultofmultiplelinearregressionanalysis.docx

- Table2TherelationshipbetweenMICandtoothloss.docx

- Table1Thedescriptionofthesubjects.docx 